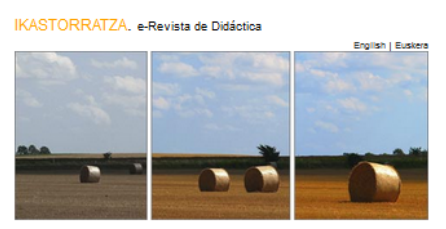

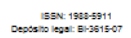

Inicio, Sobre nosotros, Publicacionesı, Participa

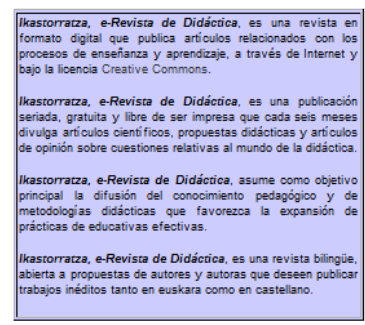

\section{IKASTORRATZA. Didaktikarako e-aldizkaria}

\author{
IKASTORRATZA. e-journal on Didactics
}

ISSN: 1988-5911 (Online) Journal homepage: http://www.ehu.eus/ikastorratza/

\title{
Efectividad de pautas de actuación para una tutoría ABP
}

\author{
Araitz Uskola \\ araitz.uskola@ehu.eus \\ Ana Arribillaga \\ ana.arribillaga@ehu.eus \\ José $\mathbf{M}^{\mathrm{a}}$ Madariaga \\ josetxu.madariaga@ehu.eus \\ Gurutze Maguregi \\ gurutze.maguregi@ehu.eus \\ $M^{a}$ Dolores Fernández \\ lola.fernandez@ehu.eus
}

To cite this article:

Uskola, A., Arribillaga, A., Madariaga, J.M., Maguregi, G., \& Fernández, M.D. (2020). Efectividad de pautas de actuación para una tutoría ABP. IKASTORRATZA. e-Revista de Didáctica, 24, 41-60. DOI: 10.37261/24_alea/3

To link to this article:

https://doi.org/10.37261/24_alea/3

Published online: 30 Abril 2020 


\section{Efectividad de pautas de actuación para una tutoría ABP}

Araitz Uskola

Matematika eta Zientzia Esperimentalen Didaktika Saila Universidad del País Vasco/Euskal Herriko Unibertsitatea (UPV/EHU), Leioa.

araitz.uskola@ehu.eus

Ana Arribillaga

Bilakaeraren eta Hezkuntzaren Psikologia Saila Universidad del País Vasco/Euskal Herriko Unibertsitatea (UPV/EHU), Leioa. ana.arribillaga@ehu.eus

José $\mathbf{M}^{\mathrm{a}}$ Madariaga

Bilakaeraren eta Hezkuntzaren Psikologia Saila Universidad del País Vasco/Euskal Herriko Unibertsitatea (UPV/EHU), Leioa. josetxu.madariaga@ehu.eus

Gurutze Maguregi

Matematika eta Zientzia Esperimentalen Didaktika Saila. Universidad del País Vasco/Euskal Herriko Unibertsitatea (UPV/EHU), Leioa gurutze.maguregi@ehu.eus

$M^{\mathrm{a}}$ Dolores Fernández

Matematika eta Zientzia Esperimentalen Didaktika Saila. Universidad del País Vasco/Euskal Herriko Unibertsitatea (UPV/EHU), Leioa lola.fernandez@ehu.eus 


\section{Resumen}

La actuación del tutor en las tutorías grupales en el Aprendizaje Basado en Problemas ha sido escasamente investigada. En este trabajo se investigan las intervenciones de tres tutores y sus 43 estudiantes en una primera sesión de tutoría ABP. Los tutores habían recibido recomendaciones, basadas en resultados de investigaciones previas, para la sesión (contextualizar la tarea, dinamizar el grupo, impulsar el aprendizaje y solicitar al alumnado la fundamentación de sus opiniones). Se han transcrito y categorizado las intervenciones de tutoras y estudiantes, realizando un análisis de su contenido. Las categorías están basadas en los referentes teóricos. Se analiza el grado de implementación de las pautas recomendadas para los tutores y la efectividad en la participación del alumnado y en su producción y elaboración de ideas. Los resultados muestran que la mayoría de las pautas se han implementado, ya que los tutores incrementan las intervenciones de los tipos recomendados. Aumenta la participación de las y los estudiantes, que generan ideas relacionadas con los objetivos de aprendizaje. Además, se observa que las ideas son altamente compartidas y elaboradas. Se concluye que las pautas recomendadas han sido efectivas para la primera sesión de tutoría ABP. Se discute acerca del papel de la reflexión de los tutores sobre la propia práctica y su influencia en la interiorización e implementación de las pautas de actuación.

\section{Abstract}

The acting of the tutor in tutoring sessions in groups during Problem Based Learning has been scarcely studied. In this study the interventions of three tutors and their 43 students in a first PBL tutoring session are analysed. The tutors had taken some recommendations for acting during the session, based on the results of previous research. Those guidelines included contextualizing the task, dynamizing the group, fostering learning and asking for evidence to students. The sessions have been audiotaped and videotaped and afterwards transcribed. The interventions have been categorized, making a content analysis. The categories are theoretically grounded. It is studied how three tutors act, how they implement previously recommended guidelines and which is the effectiveness in students' participation, generation and elaboration of ideas. The results show that tutors increase the interventions of the recommended types. The participation of the students is high, they pose ideas related to the learning-outcomes, and they share and elaborate ideas. It is concluded that the recommended guidelines have been effective for the first tutoring 
session of PBL. It is discussed that the role of the reflection of the tutors about their own professional practice has been key for adopting or not effective guidelines.

Palabras clave: Aprendizaje Basado en Problemas; Formación de profesores; Papel del profesor; Tutoría.

Key words: Preservice teacher; Problem Based Learning; Teacher role; Tutoring. 


\section{Introducción}

La formación de maestros y maestras debería, según Esteban y Mellen (2016), modificarse para que facilitara una transformación personal de los estudiantes, y para que se fomentara un saber humano. En línea con esta visión, en el centro en el que trabajan los autores, se ha querido superar la concepción de asignaturas cerradas y dar una visión de conjunto, al tiempo que se posibilitaba trabajar sobre temas sociales y culturales ligados a la profesión de maestro/a. Para ello, el Grado de Educación Primaria está organizado de forma que cada cuatrimestre el alumnado debe realizar una Tarea Interdisciplinar de Módulo (TIM), desde la perspectiva de las diferentes materias del cuatrimestre.

Para realizar esta tarea, el alumnado se organiza en grupos, tutorizados por un miembro del equipo docente. La metodología elegida es el Aprendizaje Basado en Problemas (ABP). La función de los tutores consiste en facilitar este proceso, teniendo en cuenta que ninguno es “experto” en el problema, sino en una de las materias implicadas (Dolmans et al., 2002). Las tutorías son claves para el proceso de aprendizaje del alumnado y para que no sean meras “ventanillas de reclamaciones” (Esteban y Mellan, 2016, p. 192), se han diseñado y dotado de contenido (Uskola et al., 2015). Sin embargo, las dinámicas e intercambios que surgen en dichas tutorías resultan en ocasiones tan importantes como desconocidas, por lo que surge el interés de conocerlas y mejorarlas (Chi et al., 2001; Chng et al., 2011; Maudsley, 1999, 2003; Zhang et al., 2010).

En un estudio previo (Uskola et al., 2018), se pudo comprobar que en los grupos de estudiantes de más de 10 miembros, en el grupo en que la tutora apenas intervino, pocos estudiantes participaron, se generaron pocas ideas y éstas fueron meramente citadas, sin posterior discusión o elaboración; sin embargo, en el grupo en que la tutora había realizado un mayor número de intervenciones de dinamización, hubo mayor participación del alumnado, surgieron más ideas y éstas fueron compartidas entre más estudiantes. Como consecuencia, se formuló la hipótesis de que determinadas pautas de actuación podían facilitar mejores resultados en el alumnado. Junto con un asesor externo (Branda, comunicación personal, 2014) se elaboraron unas pautas de actuación para los tutores: a) contextualizar claramente la tarea a realizar, al inicio de la sesión; b) aumentar las intervenciones dirigidas a la dinamización del grupo; c) aumentar las intervenciones dirigidas al impulso del aprendizaje, así como d) pedir al alumnado la fundamentación de 
sus opiniones a través de evidencias y datos. En este trabajo se pretende valorar la efectividad de dichas pautas de actuación.

\section{El Aprendizaje Basado en Problemas (ABP)}

El ABP comenzó a desarrollarse en la Facultad de Medicina de la Universidad de McMaster en Canadá en la década de los 60 (Branda, 2001), donde el curriculum se organizó en base al planteamiento de diferentes situaciones problemáticas contextualizadas en la vida real que permitían relacionar diferentes áreas de conocimiento. Actualmente, este método es utilizado como estrategia didáctica en otros campos de conocimiento de la educación universitaria, adoptando diferentes perspectivas (Savery, 2006). Este trabajo se sitúa en línea con el modelo de McMaster.

El ABP se compone de tres fases: análisis del problema, aprendizaje autodirigido y colaborativo, y presentación de informes (Chng et al., 2011). Durante la primera fase, el alumnado examina el problema en grupo y trata de darle explicaciones basándose en sus conocimientos previos, al mismo tiempo que identifica los interrogantes que debe responder para poder entender o resolver el problema planteado.

Hay tres elementos esenciales en la metodología ABP: los problemas, el alumnado y los tutores. Desde esa perspectiva, la función de los tutores es la de, actuar como facilitadores o guías del proceso de construcción de conocimiento, ayudando al alumnado a relacionar su conocimiento previo sobre el problema con el conocimiento existente, a integrar diferentes perspectivas y principios científicos, a desarrollar el pensamiento crítico (Branda, 2011). Asociada a esta función, proporcionan oportunidades para que se produzca el discurso constructivo, garantizan el funcionamiento eficaz del grupo y, en definitiva, facilitan el proceso de construcción de conocimiento en todos los estudiantes (AlHoqail y Badr, 2010; Morales y Landa, 2004).

\section{Hacia una tutorización más facilitadora}

Existen distintos modelos de tutorización, (Hmelo-Silver y Barrows, 2006; Schmitdt y Moust, 2000). Dolmans et al. (2002) investigaron acerca de cuáles son más propios de los tutores “expertos” y “no expertos” en el contenido. Estos estudios avalaron la idea de que, el tutor “no experto” utiliza más la congruencia social, vehicula mucho mejor una postura no dirigista, al tiempo que favorece la autonomía del alumnado. No ser expertos en el 
contenido, parece que hace que se centren más en la facilitación del proceso, logrando así una interacción más empática con el alumnado.

Como en las sesiones de tutoría de un proceso ABP el alumnado es el que debe plantear cuestiones y construir conocimiento, la discusión de ideas es clave. El análisis del discurso analizado por Cazden (1991) muestra que, aunque no sea el estilo interactivo más habitual en las clases, las interacciones cooperativas entre iguales son las que facilitan la resolución de los problemas. Ello nos lleva a reflexionar acerca de cómo debería intervenir el tutor facilitador de forma que se produzcan dichas interacciones cooperativas y que éstas sean productivas. Branda y Lee (2000) clasificaron las intervenciones del tutor o tutora en seis categorías (Dirige, Informa, Confronta, Desafía, Educa y Comparte) situándolas en un continuo que va desde lo que denominaron tutorización jerárquica hasta la tutorización facilitadora. Y, si bien reconocieron que distintas situaciones requieren distintas intervenciones, concluyeron que las tres últimas son las más deseables para facilitar el aprendizaje autónomo de los estudiantes.

No son muchos los estudios que han analizado con detalle las intervenciones del tutor en las sesiones de tutoría en un proceso ABP (Zhang et al., 2010), aunque sí hay estudios en otros contextos. Por ejemplo, Chi et al. (2001) realizaron un exhaustivo estudio sobre tutorías individuales en las que los estudiantes participaban en una sesión de hora y media sobre el sistema circulatorio humano. En una primera fase de la investigación analizaron las intervenciones de los tutores y de los estudiantes y hallaron que las intervenciones más interactivas favorecían más el aprendizaje, y que algunas intervenciones (incluyendo las explicaciones) generaban respuestas poco profundas y aprendizaje poco profundo. En una segunda fase dieron pautas a los tutores para que no dieran explicaciones ni información y para que invitaran al diálogo y a dar respuestas constructivas, obteniendo más respuestas profundas en el alumnado.

La relevancia de las preguntas metacognitivas abiertas fue hallada también en el contexto de un proceso ABP en estudiantes de Medicina por Hmelo-Silver y Barrows (2008). En el análisis de las sesiones de tutoría, se observó que las preguntas del tutor que predominaban eran las referidas a la dinámica del grupo y a clarificar cuestiones (75\%). Consideraron que esta estrategia creaba un clima que facilitaba el aprendizaje colaborativo. Zhang et al. (2010) contribuyeron a desarrollar un modelo de análisis de las tutorías grupales en ABP y lo hicieron en un contexto de formación de profesorado, en el que participaron 35 profesores/as con un grupo de facilitadores de gran experiencia. 
Hallaron que las intervenciones que aparecían en mayor medida eran las que se dirigían al re encuadre de ideas y al replanteamiento de preguntas, y concluyeron que no existe un único modelo de facilitación exitosa, sino que debe adaptarse al tipo de alumnado y a su grado de conocimiento previo del proceso ABP.

\section{Objetivos de investigación}

El objetivo de este estudio es analizar varias tutorizaciones ABP con el fin de valorar la efectividad que tienen determinadas pautas de actuación del tutor sobre el proceso de aprendizaje del alumnado. Esta investigación se centra en la primera fase del proceso ABP, en la que el alumnado examina el problema en grupo y trata de explicarlo basándose en sus conocimientos previos, al tiempo que identifica los interrogantes que debe responder para entender o resolver el problema planteado.

Se considerará que las pautas son efectivas cuando el alumnado participa, genera un alto número de ideas, y cuando las ideas son compartidas y elaboradas entre los estudiantes. Con el fin de definir el objetivo de investigación se concreta en las siguientes preguntas:

1. ¿¿De qué manera han puesto los tutores en marcha las pautas de actuación propuestas?

2. ¿De qué manera ha actuado el alumnado en cuanto a la generación y elaboración de ideas?

\section{Metodología}

Se ha adoptado un diseño de investigación de estudio de caso basado en la investigación interpretativa (Erickson, 1989). Los investigadores han analizado los vídeos grabados durante la primera sesión de tutoría ABP. La sesión de tutoría constaba de varias fases. Para este trabajo se ha tenido en cuenta la fase de planteamiento de interrogantes posterior a la lectura del escenario, que ha durado entre 16 y 28 minutos. El análisis se ha centrado en el contenido del discurso oral tanto de las tutoras como de los estudiantes. Tanto en lo referente a la primera pregunta de investigación como a la segunda, cada sesión ha sido analizada independientemente por dos investigadores para dar validez al análisis (Gee, 1999), llegando a acuerdos en los casos de interpretaciones diferentes (menos del 20\%). 


\subsection{Participantes}

El profesorado cuyas tutorías han sido analizadas pertenece a diferentes áreas de conocimiento, y ha recibido formación y asesoría sobre metodología ABP y tutorización facilitadora. Este trabajo de investigación se centra en tres grupos de estudiantes (14-15 estudiantes en cada grupo, 43 en total), tutorizados cada uno por una tutora, en febrero del curso 2014/15.

Se ha denominado a las tres tutoras analizadas como T3, T4 y T5 y al alumnado como A precedido de la tutora correspondiente. T3 tutorizó a 14 estudiantes (de T3A1 a T3A14), T4 tutorizó a 14 estudiantes (de T4A1 a T4A14) y T5 tutorizó a 15 estudiantes (de T5A1 a T5A15).

A efectos de análisis de resultados, se realiza la comparación con los grupos tutorizados por T3 (16 estudiantes) y T4 (14 estudiantes) durante el curso 2013/14.

\subsection{Procedimiento de análisis}

\subsubsection{Puesta en marcha de las pautas de actuación}

Para el análisis de resultados, se ha transcrito y analizado el contenido de todos los turnos de palabra de las tutoras. El instrumento desde que se analizaron y categorizaron los turnos de palabra han seguido el modelo de categorías determinadas en un estudio anterior (Uskola et al., 2018). Así, la categoría A recoge intervenciones referidas a la contextualización. Las de tipo B se refieren a la presentación de la tarea. Las que tienen como finalidad la dinamización del grupo fueron denominadas intervenciones tipo $\mathrm{C}$ y las que persiguen el impulso del aprendizaje, tipo D. Hay otras cuatro categorías, que no se analizan en este trabajo.

Dado que las pautas de actuación recomendadas se relacionan fundamentalmente con aumentar las intervenciones de tipo C y D, para este estudio se ha afinado el análisis de estas intervenciones, tratando de establecer subtipos o subcategorías. De esta manera, las de tipo $\mathrm{C}$ se han dividido en 3 subtipos (C1, C2, C3), según el grado de concreción o dirigismo del tutor (Branda y Lee, 2000). En la tabla 1 se muestran explicaciones y ejemplos de intervenciones de cada subcategoría.

En el caso de de las intervenciones de tipo D, se han identificado 6 subcategorías. Dos de ellas recogen intervenciones de devolución de las ideas expresadas (D1 y D2). Las otras cinco subcategorías son las que en la taxonomía de Anderson y Krathwohl (2001) corresponderían con el dominio cognoscitivo. Para establecerlas, se han considerado los 
niveles que los autores establecen dentro del citado dominio: conocimiento, comprensión, aplicación, análisis, síntesis o creación y evaluación o capacidad crítica.

Tabla 1. Subcategorías del tipo C.

\begin{tabular}{|c|c|c|}
\hline Subcategoría & Explicación & Ejemplos \\
\hline \multirow[t]{2}{*}{$\mathrm{C} 1$} & $\begin{array}{l}\text { Se invita al alumnado a participar, } \\
\text { de manera general }\end{array}$ & $\begin{array}{l}\text { "Venga, más opiniones diferentes." (T3, } \\
\text { turno } \mathrm{n}^{\circ} 6 \text { ) }\end{array}$ \\
\hline & & $\begin{array}{l}\text { “Empezamos?, ¿quién se anima?” (T4, } \\
\text { turno } \mathrm{n}^{\circ} 1 \text { ) }\end{array}$ \\
\hline \multirow[t]{2}{*}{$\mathrm{C} 2$} & $\begin{array}{l}\text { Se señala que algunos estudiantes } \\
\text { no han participado }\end{array}$ & $\begin{array}{l}\text { "Algunos no han hablado aún” (T3, turno no } \\
\text { 29) }\end{array}$ \\
\hline & & “¿Qué pensáis por ahí?” (T5, turno nº 47) \\
\hline \multirow[t]{2}{*}{$\mathrm{C} 3$} & $\begin{array}{l}\text { Se apela directamente a alguna } \\
\text { persona que no ha participado }\end{array}$ & $\begin{array}{l}\text { “Tú, ¿cómo ves la situación?” (T4, turno } \mathrm{n}^{\circ} \\
\text { 91) }\end{array}$ \\
\hline & & “¿Tú? (T5, turno n 77) \\
\hline
\end{tabular}

En la tabla 2 se muestran ejemplos y la correspondencia de las categorías con los niveles propuestos por Anderson y Krathwohl (2001).

Tabla 2. Subcategorías del tipo D.

\begin{tabular}{|c|c|c|}
\hline Subcategoría & Explicación & Ejemplos \\
\hline D1 & $\begin{array}{l}\text { Se devuelve una idea que ha } \\
\text { aparecido }\end{array}$ & $\begin{array}{l}\text { “Entonces, la clase social, la imagen, ¿qué? (T3, } \\
\left.\text { turno } \mathrm{n}^{\circ} 39\right)\end{array}$ \\
\hline D2 & $\begin{array}{l}\text { Se pregunta acerca de la } \\
\text { opinión sobre una idea ya } \\
\text { expresada }\end{array}$ & $\begin{array}{l}\text { ¿Los demás qué pensáis? A5 ha señalado dos } \\
\text { cosas, que salir en la tele y ser caro convierten al } \\
\left.\text { producto en mejor” (T4, turno } \mathrm{n}^{\circ} 7\right)\end{array}$ \\
\hline $\begin{array}{l}\text { D3 } \\
\text { conocimiento }\end{array}$ & $\begin{array}{l}\text { Se solicita explicación de lo } \\
\text { dicho }\end{array}$ & $\begin{array}{l}\text { “Respetar a los demás...¿qué significa eso?” } \\
\left(\mathrm{T} 3, \text { turno } \mathrm{n}^{\circ} 113\right)\end{array}$ \\
\hline $\begin{array}{l}\text { D4 } \\
\text { aplicación }\end{array}$ & $\begin{array}{l}\text { Se pide profundización y } \\
\text { cuestionamiento de lo dicho }\end{array}$ & $\begin{array}{l}\text { "Han salido muchas ideas...ayudad a } \\
\left.\text { completar" (T5, turno } \mathrm{n}^{\circ} 35\right)\end{array}$ \\
\hline $\begin{array}{l}\text { D5 } \\
\text { aplicación y } \\
\text { análisis }\end{array}$ & $\begin{array}{l}\text { Se demanda establecer } \\
\text { relaciones entre conceptos }\end{array}$ & $\begin{array}{l}\text { ¿De acuerdo?, “¿en desacuerdo?”, ¿con otras } \\
\text { cosas ocurre también lo mismo?” (T4, turno } \mathrm{n}^{\circ} \\
32)\end{array}$ \\
\hline $\begin{array}{l}\text { D6 } \\
\text { análisis y } \\
\text { capacidad } \\
\text { crítica }\end{array}$ & $\begin{array}{l}\text { Se requieren evidencias en } \\
\text { las que sustentar las } \\
\text { opiniones }\end{array}$ & $\begin{array}{l}\text { “Tenemos alguna evidencia para afirmar lo que } \\
\text { dices?, ¿habéis vivido las dos situaciones?” (T4, } \\
\text { turno } \mathrm{n}^{\circ} \text { 95) }\end{array}$ \\
\hline
\end{tabular}




\subsubsection{Actuación del alumnado}

Se ha procedido a transcribir todos los turnos de palabra de los estudiantes, categorizando las ideas tratadas según su relación con los objetivos de aprendizaje. Así, se cuantificó cuántos estudiantes participan (primer indicador), cuántas ideas se generan (segundo indicador), cuántos estudiantes debaten acerca de cada idea (tercer indicador). Se han comparado los resultados con los de la investigación anterior (Uskola et al., 2018).

Además, en este trabajo se ha considerado interesante profundizar este análisis, diferenciando las ocasiones en las que las ideas eran meramente mencionadas de las ocasiones en que las ideas se elaboraban (cuarto indicador), bien explicándolas, buscando sus causas, relacionándolas con otras o aportando evidencias. Para analizar si una idea era meramente citada o se elaboraba, el turno de palabra no se ha considerado una unidad adecuada, ya que las ideas se elaboran a lo largo de varios turnos. Debido a ello, se ha segmentado la sesión en distintos episodios que constituyen las unidades básicas de análisis (Lemke, 1998). Un episodio es un ciclo de turnos iniciado por la tutora en el que se mantiene la dinámica del grupo. Hay episodios en que el grupo esté barajando varias ideas y episodios en que esté elaborando una única idea.

\section{Resultados}

En primer lugar, se presenta una descripción general de lo ocurrido en las sesiones objeto de estudio. A continuación, se presentan los resultados referidos a las dos preguntas de investigación.

En la Figura 1 se han representado, para las tres tutorizaciones, todos los turnos de palabra habidos en cada grupo (eje de abscisas). En el eje de ordenadas, se han diferenciado los turnos según se traten de intervenciones de estudiantes (nivel base), intervenciones de la tutora de tipos distintos a C o D (nivel 1), intervenciones de la tutora de tipo C (nivel 2), intervenciones de la tutora de subtipos D1 y D2 (nivel 3), e intervenciones de la tutora de subtipos D3 a D6 (nivel 4). En la Figura 1 se aprecia que T4 se mantiene más tiempo en silencio durante las intervenciones del alumnado que las otras dos tutoras. Así, T4 realiza un $28 \%$ del total de turnos, mientras que T3 un 46\%, y T5 un $40 \%$.

T3 inicia la tutorización con intervenciones de tipo C, alternando sus intervenciones con las del alumnado. Este tipo de intervenciones se mantienen durante tres episodios para pasar seguidamente a intervenciones que tratan de facilitar el proceso de aprendizaje (tipo D). T4 desde los primeros momentos realiza intervenciones de los subtipos D3 a D6, 
demandando un mayor nivel de elaboración en la respuesta al alumnado. T5 interviene con tipos C y D desde el inicio, y mantiene su estrategia, alternando subtipos.

Figura 1. Representación de todos los turnos de palabra de la primera sesión ABP de tres tutoras (T3, T4 y T5). Se indican los episodios (E).

T3

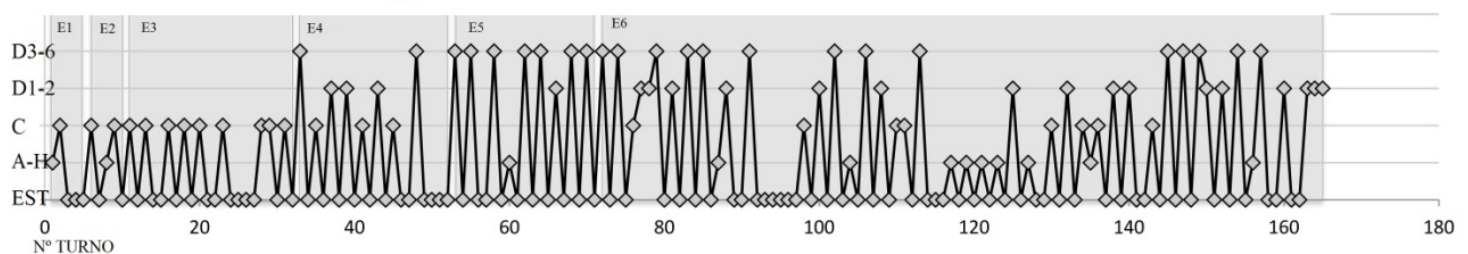

T4

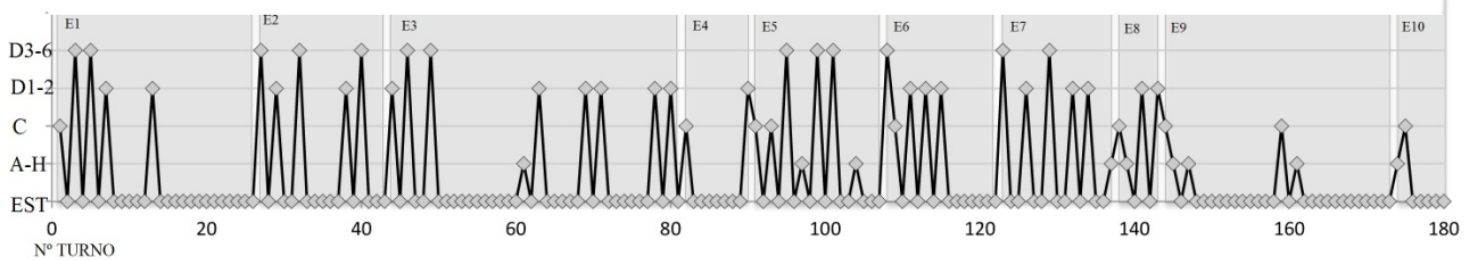

T5

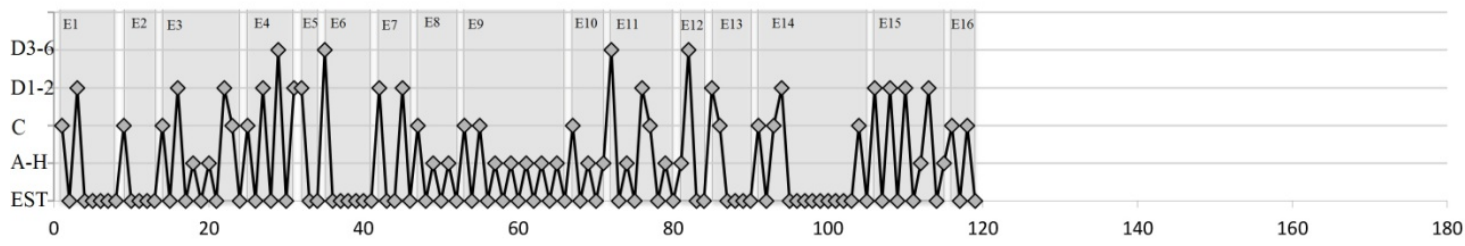

\subsection{Puesta en marcha de las pautas de actuación}

El tiempo empleado para el debate por parte de las tutoras fue de entre 16 y 29 minutos (promedio de 23). En cuanto a los tipos de intervenciones, se muestran en la Figura 2.

Figura 2. Número de intervenciones de cada tipo en la primera sesión ABP de tres tutoras (T3, T4 y T5) en los cursos 2013/14 y 2014/15

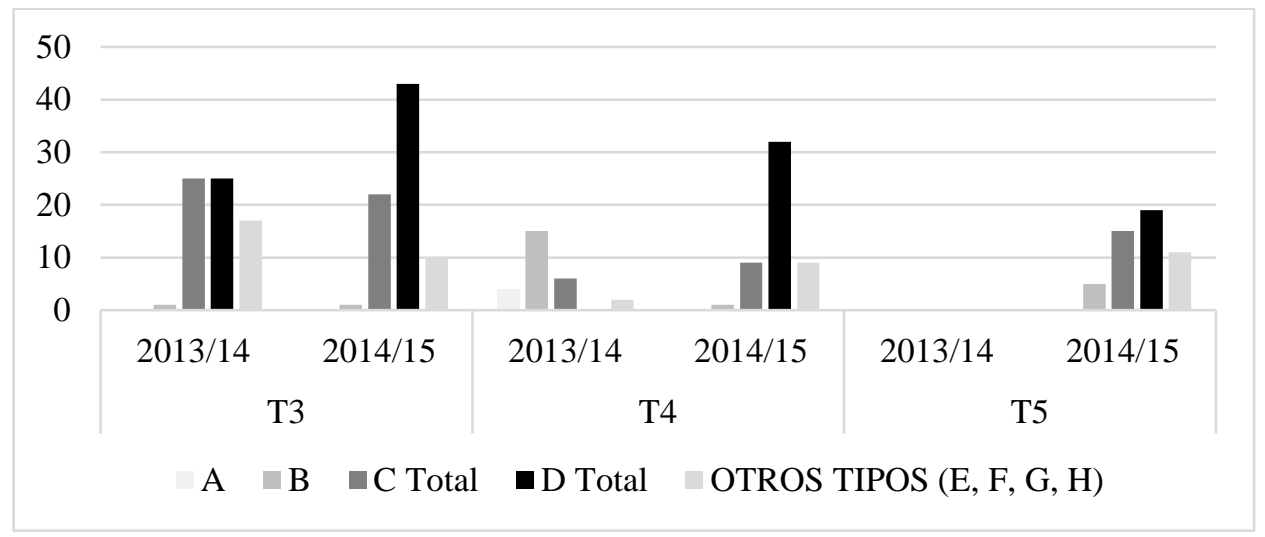


Como se observa en la Figura 2, tanto T3 como T4 aumentan en el curso 2014/15 el número de intervenciones, especialmente T4, que casi las dobla. El número de intervenciones de T5 es similar al de T4, y aunque T5 declara que en 2013/14 realizó un número muy inferior de intervenciones, la falta de datos impide establecer comparaciones cuantitativas.

A continuación, se presentan los resultados para cada pauta de actuación propuesta.

a) Contextualizar claramente la tarea a realizar, al inicio de la sesión

Dado que se está analizando la fase de la sesión posterior a la contextualización, los datos que se tienen en cuenta son las intervenciones de la tutora a demanda del alumnado, que se han diferenciado dentro de las de tipo A y B. Los resultados muestran que se ha realizado un menor número de intervenciones de este tipo, de las 15 que tenían en 2013/14 T3 y T4 se ha pasado a ninguna en 2014/15. Solo T5 interviene tres veces en 2014/15 a petición del alumnado.

b) Aumentar intervenciones dirigidas a la dinamización del grupo

En cuanto a las intervenciones de dinamización del grupo (tipo C), como se observa en la Figura 2, no hay diferencias significativas en T3, que ya en 2013/14 realizó un alto número de intervenciones de este tipo. Hay un aumento en T4, que pasa de 6 a 9 intervenciones durante el 2014/15. En el caso de T5 son 15 las intervenciones (un 30\%).

En la Figura 3 se muestra el porcentaje de intervenciones de tipo C de cada subtipo.

Figura 3. Porcentaje de cada subtipo de intervenciones $C$ utilizado en la primera sesión ABP por tres tutoras (T3, T4 y T5) en 2014/15

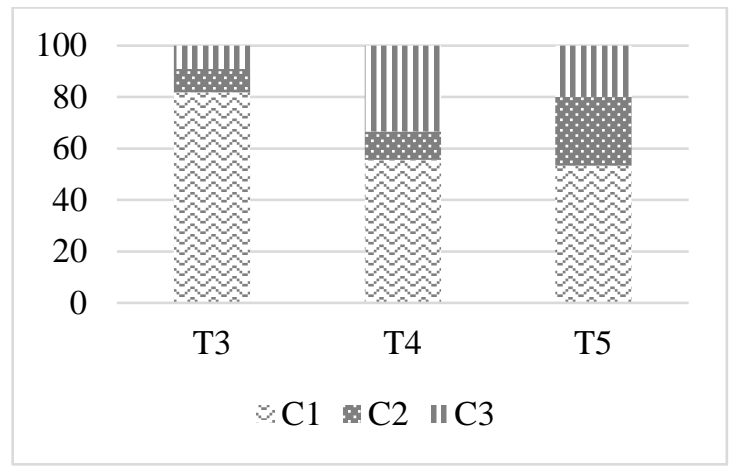

En la Figura 3 se puede ver que mientras que T3 interviene sobre todo invitando al alumnado a participar en la lluvia de ideas de manera general (el 82\% de sus intervenciones de tipo C son de subtipo C1), T4 y T5 lo hacen de forma más concreta (en torno al 50\% de intervenciones del subtipo C1 y 50\% del resto). 


\section{c) Aumentar intervenciones dirigidas al impulso del aprendizaje}

Las intervenciones con la finalidad de facilitar el proceso de aprendizaje del alumnado, de tipo D, son las más numerosas en todos los casos en el curso 2014/15, como puede verse en la Figura 2. Más de la mitad del total de las intervenciones son de este tipo en el caso de T3 (57\%) y T4 (63\%). En el caso de T5 (38\%) son también las más numerosas respecto al total.

En cuanto a las subcategorías, éstas se muestran en la Figura 4.

Figura 4. Porcentaje de cada subtipo de intervenciones D utilizado en la primera sesión ABP por tres tutoras (T3, T4 y T5) en 2014/15

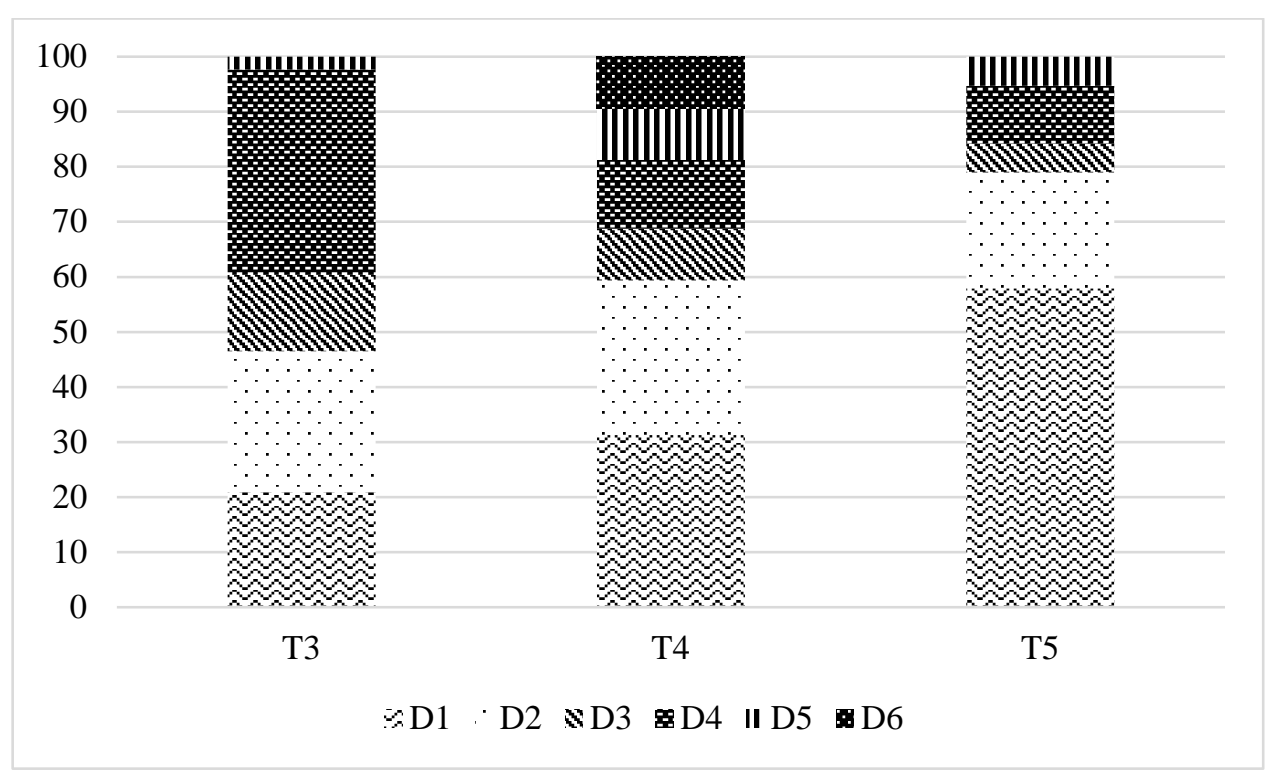

Como se ve en la Figura 4, las de tipo D1 y D2 representan la mayoría (47\% para T3, 59\% para T4 y 79\% para T5, respecto del conjunto de las D). Del conjunto D3-D6, las más numerosas son las de subtipo D4, en las que la tutora pide profundización en las ideas. Las de subtipo D5 y D6 son escasas.

d) Pedir al alumnado la fundamentación de sus opiniones a través de evidencias o datos Como se observa en la Figura 4, sólo T4 realiza intervenciones de subtipo D6, relacionadas con esta pauta de actuación, solicitando hasta en tres ocasiones evidencias o pruebas.

\subsection{Actuación del alumnado}

En la Tabla 3 se muestran las ideas mencionadas por los estudiantes en 2013/14 y en 2014/15. 
Tabla 3. Intervenciones del alumnado en los cursos 2013/14 y 2014/15. Se indican en negrita las ideas citadas por cinco o más estudiantes

\begin{tabular}{|c|c|c|c|c|c|c|}
\hline \multirow[b]{2}{*}{ CURSO } & \multicolumn{2}{|c|}{ T3 } & \multicolumn{2}{|c|}{$\mathrm{T} 4$} & \multicolumn{2}{|c|}{$\mathrm{T} 5$} \\
\hline & $2013 / 14$ & $2014 / 15$ & $2013 / 14$ & $2014 / 15$ & 2013/14 & $2014 / 15$ \\
\hline $\mathrm{N}^{\mathrm{o}}$ total de alumnas/os & 16 & 14 & 14 & 14 & & 15 \\
\hline $\mathrm{N}^{\mathrm{o}}$ alumnas/os que participan & 16 & 13 & 6 & 13 & & 12 \\
\hline 1 Educación, escuela & 14 & 13 & 5 & 5 & & 5 \\
\hline 2 Género & 1 & 3 & 2 & 10 & & 2 \\
\hline $\begin{array}{l}3 \text { Publicidad, medios de } \\
\text { comunicación }\end{array}$ & 3 & 4 & 3 & 5 & & 2 \\
\hline $\begin{array}{l}4 \text { Autoestima y pertenencia al } \\
\text { grupo }\end{array}$ & 7 & 0 & 2 & 9 & & 3 \\
\hline $\begin{array}{l}5 \\
\text { consumismo }\end{array}$ & 6 & 0 & 0 & 7 & & 7 \\
\hline 6 Importancia de las marcas & 7 & 2 & 1 & 4 & & 1 \\
\hline 7 Calidad & 2 & 2 & 0 & 4 & & 1 \\
\hline 8 Apariencias, imagen & 5 & 3 & 0 & 5 & & 4 \\
\hline 9 Edad & 5 & 2 & 1 & 7 & & 5 \\
\hline 10 Influencia de la familia & 8 & 8 & 2 & 9 & & 3 \\
\hline Total ideas & 10 & 8 & 7 & 10 & & 10 \\
\hline
\end{tabular}

En relación al primer indicador, la participación del alumnado, se observa (Tabla 3) que en el curso 2014/15 han participado casi un 90\% de los estudiantes y que la participación no ha sido menor del $80 \%$ en ningún grupo, mientras que en uno de los grupos del curso 2013/14 fue del $43 \%$.

En cuanto al segundo indicador, esto es, el número de ideas que aparecen en la sesión, se observa (Tabla 3) que en el curso 2014/15 los estudiantes del grupo de T3 han planteado ideas en el $80 \%$ de grupos de ideas relacionadas con los objetivos de aprendizaje y en los grupos de T4 y T5 en el 100\%. La diferencia respecto a 2013/14, aunque positiva, es mínima, pasando de un $85 \%$ a un $93 \%$ en el conjunto de tutoras.

Si se toma como criterio la participación de cinco o más estudiantes para establecer que una idea es compartida entre varios, globalmente no hay grandes cambios entre 2013/14 y 2014/15, con una proporción cercana al 50\% de ideas compartidas por 5 o más 
estudiantes en ambos cursos. Sin embargo, hay variaciones según el grupo. Así, en el caso de T3, la situación respecto a este indicador empeora, ya que en solo dos de las categorías de ideas participan más de 5 estudiantes el curso 2014/15 mientras que el curso anterior lo hacían en siete de ellas. En el caso de T4, su situación respecto a este indicador mejora de forma notable, ya que pasa de una única categoría altamente compartida en 2013/14 a ocho, y en ningún caso tiene a menos de cuatro estudiantes discutiendo sobre una misma idea. El alumnado de T5 presenta tres categorías altamente compartidas y solo en dos de ellas hay un único estudiante.

En cuanto al cuarto criterio, es decir, al grado en que el alumnado elabora las ideas, es decir, no se limita a señalar la idea, sino que la explica, justifica, argumenta, se observa que hay elaboración de las ideas en una proporción alta de episodios. Así, en el grupo de T3, el alumnado consigue un grado de elaboración apreciable en 4 de los 6 episodios (67\%), el de T4 en 8 de los 10 episodios (80\%) y el alumnado de T5 en 7 de los 16 episodios (44\%).

\section{Discusión y conclusiones}

Los resultados muestran que las pautas de actuación propuestas para las tutoras el curso 2014/15 se han puesto en práctica en los tres casos analizados, y que han sido efectivas para la participación y elaboración de ideas por parte de los estudiantes.

En lo que se refiere a la pregunta sobre la puesta en marcha de las pautas de actuación cabe señalar que las tutoras las han puesto en marcha de forma general, aunque lo han hecho de forma diversa, a lo largo de la sesión. Así, en los resultados se ha podido ver que T3 inicia la tutorización con intervenciones de tipo C, y que luego pasa a las de tipo D, que T4 desde los primeros momentos realiza intervenciones de los subtipos D3 a D6, demandando un mayor nivel de elaboración en la respuesta al alumnado, y que T5 interviene con tipos C y D desde el inicio, y mantiene su estrategia a lo largo de la sesión. A continuación, se discuten los resultados para cada pauta de actuación.

De las cuatro pautas establecidas, las tres primeras se han puesto en práctica de forma notable, lo que se refleja en un cambio de la actuación de las tutoras respecto al curso anterior. Así, se ha puesto en marcha la primera pauta de actuación, es decir, se ha contextualizado la tarea de forma clara al inicio de la sesión, antes de la lectura del escenario, lo que se ha reflejado en pocas intervenciones de las tutoras debidas a peticiones de aclaración por parte de los estudiantes. La puesta en práctica de la segunda 
y tercera pautas de actuación, relacionadas con la dinamización de la participación y con el impulso al aprendizaje, se ha reflejado en el alto porcentaje de intervenciones tipo C y tipo D de las tres tutoras: 86\% para T3, 80\% para T4 y 68\% para T5. En los casos en que se dispone de datos, se observa una mejoría respecto al curso anterior, tanto para T3, que tenía un 74\% de intervenciones de los tipos citados, como sobre todo para T4 que tenía un 22\%. La alta proporción de intervenciones de las tutoras de estos tipos y no otros más dirigistas o que implican dar información directa sitúan a estas tutoras en la línea indicada por Chi et al. (2001). Se observa un alto número de intervenciones relacionadas con el re encuadre de ideas y replanteamiento de preguntas, como en el estudio de Zhang et al. (2010), si bien se han hallado también destacables intervenciones que van más allá del re encuadre de ideas, y se sitúan en niveles de aplicación, análisis y capacidad crítica, según la nomenclatura de Anderson y Krathwohl (2001). De hecho, en el caso de T3 y T4 las intervenciones de mayor demanda cognitiva (Anderson y Krathwohl, 2001) de subtipos D3 a D6 suponen el 53\% y 41\% de las de tipo D (y el 25\% y 30\% del total de intervenciones) respectivamente.

Respecto a la cuarta pauta de actuación, su puesta en práctica ha sido mucho menor, y solo T4 muestra intervenciones de este subtipo (D6). Esto es, la petición explícita de evidencias por parte de las tutoras ha sido escasa; se han demandado justificaciones, pero en forma de petición de explicaciones (subtipo D3) y no explícitamente de evidencias. Esto implica una necesidad de reflexión y mejora por parte del equipo, ya que la petición explícita de evidencias a los estudiantes sobre las afirmaciones que realizan tiene como objetivo hacerles ver la necesidad de buscar tales evidencias para que puedan realizar afirmaciones discernibles de las meras opiniones, algo fundamental en el pensamiento crítico (Paul y Elder, 2006).

El porqué del menor grado de puesta en práctica de una de las pautas de actuación propuestas respecto a las otras tres puede residir en el origen de su propuesta. Las tres primeras pautas derivaron de un trabajo de investigación-reflexión del equipo del que forman parte las tres tutoras. Sin embargo, la cuarta pauta fue propuesta al equipo por un asesor externo (Branda, comunicación personal, 2014).

Respecto a la efectividad de la puesta en marcha de las pautas de actuación sobre la actuación del alumnado, los resultados muestran que su puesta en marcha ha supuesto un aumento en la participación del alumnado, así como que se generaran diversidad de ideas, y que éstas fueran compartidas y elaboradas. 
Por lo que hace referencia a la efectividad de cada una de las pautas se puede señalar que: El realizar una contextualización de la tarea al inicio de la sesión, primera pauta de actuación, se ha traducido en una disminución de intervenciones del alumnado demandando aclaraciones respecto a la tarea en esta fase de lluvia de ideas. De esta manera, las intervenciones en esta fase se han centrado en mencionar las ideas que sugería el escenario y en profundizar en ellas.

La puesta en marcha de intervenciones dirigidas a la dinamización del grupo, segunda pauta de actuación, ha hecho que participe la mayoría del alumnado. El que las tutoras sean conscientes de qué estudiantes no han participado e intervengan apelándoles (subtipos C2 y C3) ha favorecido que casi todos los estudiantes del grupo tomen parte en el intercambio de ideas.

La tercera pauta de actuación se refería a realizar intervenciones dirigidas al impulso del aprendizaje (tipo D). Estas intervenciones han propiciado que un mayor número de estudiantes participe en la elaboración de cada idea, y así, que haya una alta proporción de episodios en los que las ideas son elaboradas, justificadas, comparadas y articuladas. En el caso del número de ideas compartidas, hay que comentar el caso del grupo de T3, en que la demanda de profundización en las ideas (subtipo D4) por parte de T3, ha supuesto que un gran número de estudiantes participe en la elaboración de solo dos ideas; de hecho, a lo largo de los dos últimos episodios, que corresponden al 63\% del tiempo total y al 68\% de todos los turnos, solo se discuten esas dos ideas. Esto implica que los tutores deben permanecer en todo momento conscientes no solo del grado de elaboración de las ideas, sino de que se traten todas.

El relativo alto número de intervenciones de subtipos D3 a D6, relacionadas con el dominio cognoscitivo de Anderson y Krathwohl (2001), puede haber sido uno de los detonantes de que haya habido muchos episodios en que las ideas se hayan elaborado y profundizado en mayor grado. Realizar un análisis exhaustivo de esta relación podría ser objeto de una posterior investigación.

Como conclusión general, parece que aquellas pautas sobre las que el equipo había reflexionado, se han interiorizado y se han puesto en práctica, y se han mostrado efectivas en cuanto a la actuación del alumnado en la generación de ideas. Sin embargo, la pauta propuesta por un asesor externo no ha sido puesta en marcha de la manera deseable. Esto 
subraya la importancia de la reflexión de los docentes sobre la propia práctica profesional y la necesidad de investigar y evaluar la propia práctica.

Este trabajo ha permitido al equipo reflexionar críticamente acerca de su propia práctica e interiorizar la importancia de determinadas acciones: continuar realizando un alto número de intervenciones dirigidas a dinamizar el grupo y a impulsar el aprendizaje (Branda y Lee, 2000; Chi et al., 2001), continuar y aumentar las intervenciones de los subtipos D3 a D6, demandar explícitamente pruebas al alumnado para trabajar su capacidad crítica (Paul y Elder, 2006). Todo ello para caracterizar cómo mejorar la actuación de los tutores “no expertos” en la materia (Dolmans et al., 2002), con el objetivo de que participe la totalidad del alumnado, y de que, entre todas y todos, generen $\mathrm{y}$ elaboren ideas relacionadas con todos los objetivos de aprendizaje, objetivo último de la primera sesión de tutoría en la metodología ABP (Branda, 2001).

\section{Referencias}

Alhoqail, I. A., y Badr, F. M. (2010). Objective structured brainstorming questions (OSBQs) in PBL tutorial sessions: Evidence based pilot study. International Journal of Health Sciences, 4(2), 93-102.

Anderson, L. W., y Krathwohl, D. R. (Eds.) (2001). A Taxonomy for Learning, Teaching, and Assessing: A Revision of Bloom's Taxonomy of Educational Objectives. Boston: Pearson Education Group.

Branda, L. A. (2001). Aprendizaje basado en problemas, centrado en el estudiante, orientado a la comunidad. En Universidad de Buenos Aires (eds.), Aportes para un cambio curricular en Argentina 2001. Jornadas de Cambio Curricular de la Facultad de Medicina de la Universidad de Buenos Aires (pp. 79-101). Buenos Aires: Organización Panamericana de la Salud.

Branda, L. A. (2011). El aprendizaje basado en problemas y la genuina realidad. Diario de un tutor. Educación Médica, 14(3), 151-159.

Branda, L. A., y Lee, Y. L. (2000). Evaluación de la Competencia del Tutor. Carrera de Medicina. Bahía Blanca (Argentina): Universidad Nacional del Sur.

Cazden, C. B. (1991). El discurso en el aula. El lenguaje de la enseñanza y del aprendizaje. Barcelona: Paidós-MEC. 
Chi, M. T. H., Siler, S. A., Jeong, H., Yamauchi, T., y Hausmann, R. G. (2001). Learning from human tutoring. Cognitive Science, 25(4), 471-533.

Chng, E., Yew, E. H. J., y Schmidt, H. G. (2011). Effects of tutor-related behaviours on the process of problem-based learning. Advances in Health Sciences Education, 16(4), 491-503.

Dolmans, D. H. J. M., Gijselaers, W. H., Moust, J. H. C., De Grave, W. S., Wolfhagen, I. H. A. P., y Van Der Vleuten, C. P. M. (2002). Trends in research on the tutor in problem-based learning: Conclusions and implications for educational practice and research. Medical Teacher, 24(2), 173-180.

Erickson, F. (1989). Métodos cualitativos de investigación sobre la enseñanza. En M. Wittrock (ed.), La investigación de la enseñanza, II. Métodos cualitativos y de observación (pp. 195-301). Barcelona: Paidós.

Esteban, F., y Mellen, T. (2016). ¿Por qué quieres ser maestro?, ¿cómo es un buen maestro? Ideas para la formación universitaria. Bordón, 68(2), 185-198.

Gee, J. P. (1999). An introduction to discourse analysis: Theory and method. Londres: Routledge.

Hmelo-Silver, C. E., y Barrows, H. S. (2006). Goals and strategies of a problem-based learning facilitator. Interdisciplinary Journal of Problem-based Learning, 1(1), 21- 39.

Hmelo-Silver, C. E., y Barrows, H. S. (2008). Facilitating collaborative knowledge building. Cognition and Instruction, 26(1), 48-94.

Lemke, J. L. (1998). Analysing verbal data: Principles, methods and problems. En B. J. Fraser y K. G. Tobin (eds.), International Handbook of Science Education (pp. 1175-1189). Dordrecht: Kluwer Academic Publishers.

Maudsley, G. (1999). Roles and responsibilities of the problem based learning tutor in the undergraduate medical curriculum. British Medical Journal, 318(2), 657-661.

Maudsley, G. (2003). The limits of tutors' comfort zones with four integrated knowledge themes in a problem-based undergraduate medical curriculum (Interview study). Medical Education, 37(5), 417-423. 
Morales, P., y Landa, V. (2004). Aprendizaje Basado en Problemas. Problem-Based. Learning. Theoria, 13, 145-157.

Paul, R., y Elder, L. (2006). Critical thinking (2nd ed.). Upper Saddle River (NJ): Pearson Prentice Hall.

Savery, J. R. (2006). Overview of Problem-based Learning: Definitions and distinctions. Interdisciplinary Journal of Problem-Based Learning, 1, 9-20.

Schmidt, H. G., y Moust, J. H. C. (2000). Factors affecting small-group tutorial learning: A review of research. En D. H. Evensen y C. E. Hmelo (eds.), Problem-based learning: A research perspective on learning interactions (pp. 19-52). Mahwah (NJ): Lawrence Erlbaum.

Uskola, A., Madariaga, J. M., Arribillaga, A., Maguregi, G., y Fernández, M. D. (2018). Categorisation of the interventions of facilitating tutors on PBL and their relationship with students' response. Profesorado. Revista de currículum y formación del profesorado, 22(4), 153-170.

Uskola, A., Madariaga, J. M., Arribillaga, A., Maguregi, G., Romero, A., y Fernández, M. D. (2015). Propuesta e implementación de un plan de tutorización de una tarea interdisciplinar universitaria de carácter modular. Revista de Docencia Universitaria, 13(2), 207-235.

Zhang, M., Lundeberg, M., McConnell, T. J., Koehler, M. J., y Eberhardt, J. (2010). Using questioning to facilitate discussion of science teaching problems in teacher professional development. The Interdisciplinary Journal of Problem-based Learning, 4(1), 57-82. 